\title{
LOS SUBSISTEMAS DE OPOSICIONES FONEMÁTICAS DE LAS FRICATIVAS EN EL ESPAÑOL DE AMÉRICA
}

\author{
Annette Calvo Shadid
}

\begin{abstract}
RESUMEN
Sobre la base del concepto de presión estructural, esta investigación muestra cómo algunos cambios fonéticos dados en la serie [+obstruyente +continua] del español han repercutido en el sistema fonológico, transformando los rasgos que diferencian los fonemas entre sí en nuevos tipos de oposiciones fonológicas.

De este modo, se propone que existen al menos cinco subsistemas de oposiciones fonemáticas de fricativas en el español de América, cuyas diferentes transfonologizaciones han permitido, en algunos casos, llenar el vacío fonemático que se daba en la serie [+coronal -anterior].
\end{abstract}

\begin{abstract}
On the basis of the concept of structural pressure, this investigation shows how some phonetic changes in the spanish series of [+obstruent, -continuant] have had a repercussion on the phonologicas system, transforming the traits that differentiate the phonemes among themselves into new types of phonological oppositions.

Thus, we propound the existence of at least five subsystems of phonematic oppositions of fricatives in American spanish, whose transphonologizations have allowed, in some instances, the filling of the phonematic void left in the series [+coronal, -anterior].
\end{abstract}

\section{Introducción}

\subsection{Sistema fonológico del español de América durante el siglo XVII}

Un estudio sobre los sistemas lingüísticos sincrónicos emparentados que se base en las diversas variantes dialectales debe fundamentarse, de hecho, en los cambios estructurales diacrónicos sufridos por las variedades que se comparan.

Durante el siglo XVII, se consumó en todo el ámbito hispanoamericano una serie de cambios en el sistema de oposiciones fonemáticas que se venían gestando desde el siglo XV.

Estos cambios incluyeron la desafricación de los fonemas / ts / y / dz / $>/ \mathrm{s} / \mathrm{y} / \theta /$ y la pérdida de su distinción, el ensordecimiento de $/ \mathrm{z} / \mathrm{y} / \mathrm{z} / \mathrm{>} / \mathrm{s} / \mathrm{y} / \mathrm{s} / \mathrm{y}$, asimismo, la 
pérdida de su distinción, la posteriorización de $/ \int />/ x /$, entre otros. Todos ellos se llevan a cabo entre los siglos XVI y XVII, aunque varíen las fechas de realización según las diferentes regiones ${ }^{1}$.

Lo anterior dio como resultado el sistema fonológico moderno del español de América a partir del siglo XVII, el cual se muestra en el Cuadro 1.

\section{Cuadro 1}

Sistema fonológico máximo de consonantes en el español de América

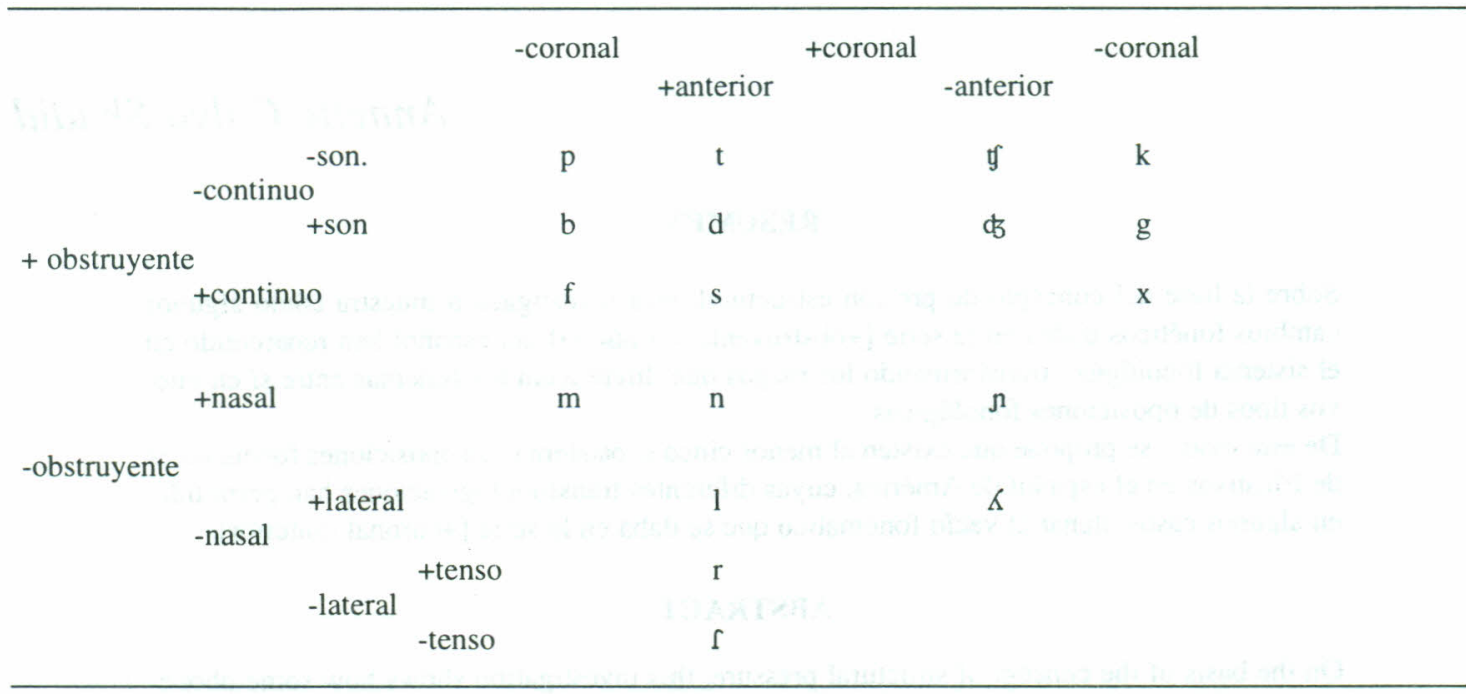

\subsection{Objetivo de la investigación}

El sistema fonológico anterior se considera la base a partir de la cual han emergido los diferentes sistemas fonológicos del español actual en América.

El presente trabajo describe los diferentes subsistemas de fricativas en las distintas variedades del español americano. Las diferencias entre los subsistemas se analizan sobre la base del concepto de presión estructural .

Como se puede observar en el Cuadro 1, el sistema original de fricativas del español presenta un vacío fonemático en la serie +coronal -anterior. De tal modo, se experimentan cambios fonéticos en esta serie de sonidos. Dichos cambios implican desplazamientos de segmentos, los cuales ejercen sobre otros ciertas presiones. De esta manera, esos otros segmentos pueden también desplazarse para alejarse de la confusión con el segmento que establece tal presión.

Cuando los cambios fonéticos repercuten en el sistema, transformando los rasgos que diferencian los fonemas entre sí, pueden darse nuevos tipos de oposiciones fonológicas.

Existen tres cambios estructurales que tienen como consecuencia una modificación en el sistema de oposiciones fonológicas en cualquier lengua ${ }^{2}$ :

Desfonologización: es una mutación fónica que implica la supresión de una diferencia fonológica. Este proceso determina una disminución en el inventario fonemático, debido a una fusión de fonemas diferentes en uno solo. 
Fonologización: consiste en la aparición de una nueva oposición distintiva en la lengua. En este caso, ocurre un aumento en el número de fonemas del sistema.

Transfonologización: una oposición fonológica existente en la lengua se transforma en otra oposición fonológica heterogénea. Este cambio no implica transformaciones en el inventario de fonemas.

\section{Estudios previos}

Algunos autores han establecido nuevos tipos de oposiciones fonológicas en los subsistemas tanto de las consonantes oclusivas como de las fricativas, nasales, semivocales y vocales. En relación con las fricativas, se han propuesto las que a continuación se citan.

\subsection{Escisión del fonema / $\mathrm{s} />/ \mathrm{s} /: / \mathrm{h} /$}

Para varias zonas de Argentina, Honsa (1965) propone la fonologización del segmento [ $\mathrm{h}$ ] a partir del fonema / s / final y menciona una posible pareja mínima: / eharxen'tina / es argentina: / esarxen'tina / esa argentina. Además, señala que el fonema / h / tendría una distribución restringida a la posición final de sílaba, donde nunca aparecería el fonema / s /.

En contra de esta teoría, se puede argumentar, en primer lugar, que la supuesta pareja mínima en realidad refleja la oposición de una sutura abierta: [eharxen'tina] es argentina $(\mathrm{VC}+\mathrm{V})$, frente a una sutura cerrada [esarxen'tina] esa argentina (VCV).

En segundo lugar, como el mismo Honsa reconoce, la distribución restringida del segmento [ $\mathrm{h}$ ] hace cuestionable su carácter de fonema independiente, sobre todo al estar en distribución complementaria con [ s ]: [ peh + a'sul ] pez azul y ['pese ] peces.

\subsection{Fonologización de / $/$}

Según Fontanella de Weinberg (1980-81), en el español de Buenos Aires, el fonema I $/$ / se desarrolló como consecuencia de la introducción de préstamos de lenguas europeas, especialmente del francés, inglés e italiano.

Este fonema vendría a llenar la casilla vacía en el orden de las obstruyentes, al cual se le adscribirían los rasgos (- anterior, + coronal) de la serie ( + continuo). Este fonema completaría el haz de correlaciones fonológicas de la serie de obstruyentes:

-cont. + cont. -cont. +cont. -cont. +cont. -cont. +cont.

$\begin{array}{lllllllll}\text { son. } & \mathrm{p} & \mathrm{f} & \mathrm{t} & \mathrm{s} & \mathrm{g} & \mathrm{f} & \mathrm{k} & \mathrm{h} \\ + \text { son } & \mathrm{b} & & & \mathrm{d} & & \text { d } & & \mathrm{g}\end{array}$


Sin embargo, estudios cuantitativos sobre el yeísmo porteño indican que el fonema / d3 / presenta el segmento [ $\int$ ] entre sus realizaciones alofónicas. El hecho de que en algunas hablas se pueda establecer un contraste distintivo entre [ $\int$ ] y [ dz ] se debe más bien a una situación de préstamo léxico, propio del contacto de lenguas, que a una verdadera reestructuración fonológica.

\subsection{Escisión del fonema / $\mathbf{r} />/ \mathrm{J} /: / \mathrm{I}_{\mathrm{O}} /$}

Sánchez Corrales (1985: 65) propone una escisión fonológica de los segmentos en cuestión. Existen tres fonos, $[\underset{0}{\mathrm{I}}],[\mathrm{I}]$ y [ S ], los cuales comparten los mismos entornos, a saber, posición intervocálica, y [ 중 y [ s ], además, se dan ambos en posición final absoluta. Por lo tanto, el autor concluye que cada uno de esos fonos corresponde a fonemas diferentes.

Observa que en [kombe' I a I I] el fono [I] está tanto en posición intervocálica como en posición final. Se trata de un fonema, con un único alófono, cuya distribución en la cadena fónica es precisamente $V$ $\mathrm{V} O \mathrm{~V}$ \#.

Sánchez Corrales concluye anotando que este nuevo fonema proviene del alófono fricativo sordo [ $\left[\begin{array}{l}\mathrm{I} \\ ]\end{array}\right]$, y que es la asimilación de la [ r ] (sic) en la secuencia [ rs ] la que provoca la escisión mencionada, al elevar a rasgo funcional lo que había sido una variante combinatoria. Sin embargo, la diferenciación de estos dos fonemas es teóricamente cuestionable, si pensamos que la distribución intervocálica del sordo está limitada a una secuencia muy baja. Según los análisis realizados por la autora de esta ponencia se trata, más bien, de alófonos en distribución complementaria.

\section{Los subsistemas de fricativas en el español de América}

Partiendo de las diferentes variantes registradas en las distintas zonas dialectales, esta investigación supone que existen al menos cinco subsistemas de fricativas en el español de América, algunos de los cuales llenan el vacío fonemático en la serie +coronal -anterior.

\subsection{Subsistema 1}

En este subsistema no ha habido cambios estructurales en relación con el sistema general de fricativas del español de América. Este está representado por $\mathrm{Cuba}^{3}$, República Dominicana $a^{4}$, México $^{5}$ (con tendencia también al subsistema $2^{6}$ ), Honduras ${ }^{7}$, El Salvador ${ }^{8}$, Nicaragua $^{9}$, Guanacaste (provincia de Costa Rica) ${ }^{10}$, Panamá ${ }^{11}$, las costas y el dialecto central andino occidental de Colombia ${ }^{12}$, Venezuela ${ }^{13}$, parte norte y oriental de Bolivia ${ }^{14}$, las costas de Ecuador ${ }^{15}$, la ciudad de Lima y las provincias costeñas de Perú ${ }^{16}$, Buenos Aires y sus zonas de influencia, y el área litoral pampeana de Argentina ${ }^{17}$. 
Cuadro 2

Subsistema 1

-coronal

f
+ coronal

$S$ -coronal

$\mathrm{x}$

\subsection{Subsistema 2}

En este subsistema ha habido una transfonologización o desplazamiento del fonema -obstruyente +tenso a la serie de fricativas. Pierde su rasgo de tensión, por lo cual desaparece su oposición +tenso, -tenso. Esto indica que se da una simplificación de rasgos que se oponen y, por otro lado, se llena el vacío fonológico de la serie +coronal -anterior. Dentro de este subsistema, existe un primer subgrupo que se caracteriza por la tendencia a la asibilación; en el segundo subgrupo, la tendencia general es a la fricación sin asibilación y, frecuentemente, a la fricación y elisión en posición final.

Cuadro 3

Subsistema 2

\begin{tabular}{|c|c|c|c|c|}
\hline & \multirow[t]{2}{*}{-coronal } & \multicolumn{2}{|c|}{ +coronal } & \multirow[t]{2}{*}{-coronal } \\
\hline & & +anterior & -anterior & \\
\hline -sonoro & f & s & I & $\mathrm{x}$ \\
\hline
\end{tabular}

Este subsistema está representado, en el primer subgrupo, por la Ciudad de México ${ }^{18}$, principalmente, (ver también nota 3), Guatemala ${ }^{19}$ (parte central), Costa Rica ${ }^{20}$, dialecto andino oriental de Colombia ${ }^{21}$ (Nariño, Cundinamarca y Bocayá ,-sureste de Colombia-), la sierra ecuatoriana ${ }^{22}$, la zona andina y amazónica de Perú ${ }^{23}$, Bolivia $^{24}$ (región andina centro y sudoccidental -La Paz, Oruro, Cochabamba, Potosí, Chuquisaca y Sucre-), Paraguay ${ }^{25}$, Argentina $^{26}$ (regiones del interior como Corrientes, Mendoza, Misiones), y Chile 27.

El segundo subgrupo está representado por Cuba, Puerto Rico, República Dominicana ${ }^{28}$, y el español de la costa de Perú ${ }^{29}$. 


\subsection{Subsistema 3}

Este subsistema tiene la particularidad de que hay un desplazamiento o transfonologización del fonema +obstruyente -continuo, pues pierde su rasgo -continuo y la sonoridad, en este caso, no va a ser distintiva. guay 31 .

Este subsistema está representado por Buenos Aires, Rosario, (Argentina) ${ }^{30}$ y Uru-

Cuadro 4

Subsistema 3

\begin{tabular}{|c|c|c|c|c|}
\hline & \multirow[t]{2}{*}{-coronal } & \multicolumn{2}{|c|}{ +coronal } & \multirow[t]{2}{*}{-coronal } \\
\hline & & +anterior & -anterior & \\
\hline -sonoro & $\mathrm{f}$ & s & $\int$ & $\mathrm{x}$ \\
\hline
\end{tabular}

\subsection{Subsistema 4}

Este subsistema presenta una transfonologización del segmento -obstruyente +lateral, pues pierde su rasgo +lateral en favor del rasgo +obstruyente.

En este subsistema se observa que la oposición pollo - poyo, se realiza [pozo] - [pojo]. Está representado por la sierra ecuatoriana ${ }^{32}$, principalmente, y Santiago del Estero (Argentina) ${ }^{33}$.

\section{Cuadro 5}

Subsistema 4

\begin{tabular}{|c|c|c|c|c|}
\hline & \multirow{2}{*}{-coronal } & \multicolumn{2}{|c|}{ +coronal } & \multirow[t]{2}{*}{-coronal } \\
\hline & & tanterior & -anterior & \\
\hline -sonoro & $\mathrm{f}$ & $\mathrm{s}$ & 3 & $x$ \\
\hline
\end{tabular}

\subsection{Subsistema 5}

En este subsistema ha habido una transfonologización o desplazamiento del fonema -obstruyente +tenso / r / a la serie de fricativas (+obstruyente). Pierde su rasgo de tensión, como en el subsistema 2, pero también pierde su rasgo coronal, para pasar a ser un segmento 
-coronal -anterior, representado por / x / en el Cuadro 6. A su vez, el fonema +obstruyente anterior, / x / se ha transfonologizado en / h /, un fonema -obstruyente -silábico y, en consecuencia, también -consonante ${ }^{34}$.

\section{Cuadro 6}

Subsistema 5

\section{-coronal}

tanterior

f +coronal

s -coronal

-anterior

$\mathrm{X}$

Este subsistema está representado por Puerto Rico, principalmente, y por la parte oriental de $\mathrm{Cuba}^{35}$.

\section{Conclusiones}

Sobre la base del concepto de presión estructural, esta investigación ha mostrado cómo algunos cambios fonéticos dados en la serie +obstruyente +continua del español han repercutido en el sistema fonológico, transformando los rasgos que diferencian los fonemas entre sí en nuevos tipos de oposiciones fonológicas.

De este modo, de acuerdo con las diferentes variantes registradas en las distintas zonas dialectales, en esta investigación se ha propuesto que existen al menos cinco subsistemas de fricativas en el español de América, cuyas diferentes transfonologizaciones han permitido, en algunos casos, llenar el vacío fonemático que se daba en la serie +coronal -anterior.

\section{Notas}

1. Cf. Alarcos (1950) y Quesada Pacheco (1986).

2. Las definiciones han sido tomadas de Dubois (1973).

3 Cf. Terrel (1976) y Vaquero (1996).

4. Cf. Vaquero (1996).

5. Cf. Lope Blanch (1996).

6. Matluck (1952) registra la asibilación de / r / con una frecuencia relativa en el Valle de México. Moreno de Alba (1972: 370) afirma que la asibilación de / r / en el español de México no es tan frecuente en el ámbito nacional. La capital es un foco irradiador del fenómeno, el cual se encuentra en expansión.

7. Cf. Canfield (1981), Lipski (1987), Quesada Pacheco (1996). 
8. Cf. Canfield (1953, 1960 y 1981), Geoffroy (1982).

9. Cf. Lacayo (1954), Quesada Pacheco (1996).

10. Cf. Arrieta (1986), Gaínza (1976), Quesada Pacheco ( ).

11. Cf. Alvarado (1972) y Quesada Pacheco (1996).

12. Cf. Flórez (1961), Montes (1996).

13. Cf. Sedano y Bentivoglio (1996).

14. Cf. Gordon (1980) afirma que, en general, los llanos presentan una articulación vibrante de / r / frente al altiplano, en donde se observa, según el autor, marcadísimo predominio de la articulación asibilada en la gran mayoría de los informantes. Ver también Coello (1996).

15. Cf. Boyd-Bowman (1953), Toscano (1962), Córdova (1996).

16. Cf. Caravedo (1990).

17. Cf. Vidal de Battini (1962), Donni de Mirande (1996).

18. Cf. Moreno de Alba (1972), Perissinotto (1972, 1975), Torreblanca (1975), Lope Blanch (1996).

19. Cf. Quesada Pacheco (1996).

20. Cf., entre otros, Chavarría Aguilar (1951), Umaña (1981), Sánchez Corrales (1985), Calvo (1996), Calvo y Portilla (en prensa).

21. Cf. Gordon (1980) y Montes (1996).

22. Cf. Boyd-Bowman (1953), Toscano (1962) y Córdova (1996).

23. Cf. Caravedo (1996).

24. Cf. Coello (1996)

25. Cf. de Granda (1982), Alvar (1996).

26. Cf. Donni de Mirande (1996).

27. Cf. Oroz (1962). Wagner (1996) afirma: ... en el país / $\mathrm{r} / \mathrm{y} / \mathrm{rr} /$ son articuladas como vibrantes o como fricativas asibiladas.

28. De acuerdo con Vaquero (1996), lo característico de las vibrantes en las Antillas es su debilitamiento articulatorio general, con tendencia a la fricación.

29. Cf. Caravedo (1996).

30. Cf. Donni de Mirande (1996).

31. Cf. Donni de Mirande (1996). 
32. Cf. Córdova (1996).

33. Cf. Donni de Mirande (1996).

34. De acuerdo con Chomsky y Halle (1968), los segmentos glotales, como [ h ], no son obstruyentes y, en consecuencia, tampoco son consonantes.

35. Cf. Vaquero (1996).

\section{Bibliografía}

Alarcos Llorach, E. 1950/83. Fonología española. Madrid: Gredos.

Alvar, M. (ed.). 1996. Manual de dialectología hispánica. Barcelona: Editorial Ariel.

Alvarado, E. 1972. El español de Panamá. Estudio fonético y fonológico. Panamá: Editorial Universitaria.

Arrieta, M. et al. 1986. "Actitudes lingüísticas hacia dos variedades de habla: Valle Central y Guanacaste". Revista de Filología y Lingüística de la Universidad de Costa Rica. 12(2): 113-28.

Boyd-Bowman, P. 1953. "Sobre la pronunciación del español en Ecuador". Nueva Revista de Filología Hispánica. 7: 221-33.

Calvo Shadid, A. 1996. "Variación fonética de / / / / y / r / en el habla culta de San José". Revista de Filología y Lingüística de la Universidad de Costa Rica. 21: 115-34.

Calvo Shadid, A. y M. Portilla. 1998. "Variantes de / f / y / r / en el habla culta de San José. Káñina. XXII (1).

Chavarría Aguilar. 1951. "The phonemes of Costa Rican Spanish". Language. 27: 248-53.

Canfield, D. L. 1953. La pronunciación del español en El Salvador. San Salvador.

1960. "Observaciones sobre el español salvadoreño". Filología. 34-57.

1981. Spanish pronunciation in the Americas. Chicago.

Caravedo, R. 1996. "Perú”. En Alvar, M. (ed.).

Chavarría Aguilar, O. 1951. "The phonemes of Costa Rican Spanish". Language. 27: 248-53.

Chomsky, N. y M. Halle. 1968. The sound pattern of English. New York: Harper \& Row. 
Coello, C. 1996. "Bolivia” En: Alvar, M. (ed.).

Córdova, C. J. 1996. "Ecuador". En: Alvar, M. (ed.).

Donni de Mirande, N. 1996. “Argentina-Uruguay". En: Alvar, M. (ed.).

Dubois, J. et al. 1973. Diccionario de Lingüística. Madrid: Alianza Editorial.

Flórez, L. 1961. "El Atlas lingüístico-etnográfico de Colombia (ALEC). Nota informativa". Thesaurus. Boletín del Instituto Caro y Cuervo. Bogotá.

Fontanella de Weinberg, M. B. 1980-81. "Habla aniñada en el español bonaerense". Boletín de Filología. Universidad de Chile.

Gaínza, G. 1976. "El español de Costa Rica: breve consideración acerca de su estudio". $R_{t}$ vista de Filología y Lingüística de la Universidad de Costa Rica, 2(2): 79-83.

Geoffroy, P. 1982. El español que hablamos en El Salvador. San Salvador: Dirección de Publicaciones del Ministerio de Educación.

Gordon, A. M. 1981. "Notas sobre la fonética del castellano en Bolivia". Actas del Sexto Congreso Internacional de Hispanistas, 349-52.

Granda, G. de. 1982. "Observaciones sobre la fonética del español en el Paraguay". Anuario de Letras. 20: 145-94.

Honsa, V. 1965. “The Phonemic systems of Argentinian Spanish”. Hispania. 48: 275-83.

Lacayo. 1954. "Apuntes sobre la pronunciación del español en Nicaragua". Hispania. 37: 267-8.

Lipsky, J. 1987. Fonética y fonología del español de Honduras. Tegucigalpa: Guaymuras.

Lope Blanch, J. 1996. "México”. En: Alvar, M. (ed.).

Matluck, J. 1952. "La pronunciación del espaañol en el Valle de México". Nueva Revista de Filología Hispánica. 6: 109-120.

Montes, J. J. 1996. “Colombia”. En: Alvar, M. (ed.).

Moreno de Alba, J. 1972. "Frecuencias de la asibilación de / r / y / rr / en México". Nueva Revista de Filología Hispánica. 21: 363-70. 
Oroz, R. 1962. "El español de Chile". En: Presente y futuro de la lengua española. Madrid.

Perissinotto, G. 1972. "Distribución demográfica de la asibilación de vibrantes en la ciudad de México". Nueva Revista de Filología Hispánica, 21: 71-9.

Sedano, M. y P. Bentivoglio. 1996. "Venezuela”. En Alvar, M. (ed.).

1996. "El español de América Central”. En Alvar, M. (ed.).

Terrel, T. "La variación fonética de / r / y / rr / en el español cubano". Revista de Filología Española. 58: 109-32.

Torreblanca, M. 1984. "La asibilación de 'r' y 'rr' en la lengua española". Hispania, 67: 614-6.

Toscano, H. 1962. "El español hablado en Ecuador". Presente y futuro de la lengua española. Madrid.

Umaña Aguiar, J. 1981. "Variable vibrants in middle-class Costa Rican spanish". Tesis de maestría. Universidad de Georgetown.

Vaquero, M. 1996. “Antillas”. En: Alvar, M. (ed.).

Vidal de Battini, B. E. 1962. "El español de la Argentina". Presente y futuro de la lengua española. Madrid.

Wagner, C. 1996. "Chile”. En: Alvar, M. (ed.). 
